\title{
The Application of Visualization in Scientific Computing (VISC) on Geotechnical Engineering
}

\author{
Ting Zhang ${ }^{1, \text { a }}$ \\ ${ }^{1}$ School of Transportation Engineering, Nanjing Tech University, Nanjing 211800, China; \\ azhangtchn@163.com
}

Keywords: Visualization, geotechnical engineering, 3D technology.

\begin{abstract}
Compared to other engineering designs, due to the complex dynamics of geological conditions and the uncertainty of geotechnical engineering conditions as well as the concealment of this project, geotechnical engineering design needs to change constantly in the process of construction, on the basis of various kinds of information mainly from the construction site. This kind of information is usually in large quantities which needs rapid process, result analysis and visual display with the aid of computers. Timely feedback to the site is necessary which can guide the construction and the optimization design. This paper reviews the application of scientific computing visualization technology in three-dimensional modeling, three-dimensional numerical simulation analysis and the three-dimensional network of rock mass structure simulation research.
\end{abstract}

\section{Introduction}

Scientific computing visualization of geological information refers to the use of the appropriate data structure to establish mathematical models of geological characteristics, and the use of computer graphics to show mathematical description in the form of 3D realistic images [1,2]. With the rapid development of computer visualization technology and virtual reality technology, as well as the deepening of the geoscientific research, engineering geological scientific computing visualization has become a hot spot in the multi-disciplinary overlapping research frontier of current mathematics geology, geotechnical engineering, engineering geology and computer science.

Because the scientific computing visualization technology is able to translate space data information into three-dimensional graphics, it is effective and intuitive to use such technology to process a large amount of data. The technology has already been widely used in the field of geotechnical engineering [3]. This paper mainly describes the application of scientific computing visualization technology in three-dimensional modeling, three-dimensional numerical simulation analysis of rock mass and the three-dimensional network of rock mass structure simulation research, thus proving that the application of visualization technology in geotechnical engineering research is of great significance.

\section{3D Modeling System of Rock Mass}

The properties of the object processed in geotechnical engineering are complicated, so it is necessary to study 3D data structure and effective modeling technology. It can fully reflect the characteristics of strata, and make it convenient for researchers to manage and operate data which is the foundation of the efficient display and the analysis of the implementation system.

\subsection{Data model}

In the study of scientific computing visualization modeling system, the data model and data structure are key problems. Most of 3D spatial data model research is based on the CAD model in the past. 3D spatial data model is about the concept and the method of 3D spatial data organization, and it reflects the space entity and the contractions between each other in the real world. Oriented spatial data model can be used as a specific kind of data models, whose main research contents are the data structure, data operation and integrity constraint conditions of the three elements. The subjects handled in the geotechnical engineering include two categories, spatial and non-spatial entity, and non-spatial entity can be dealt with by using the traditional database management system. Spatial entity has spatial 
descriptions, including absolute position (such as space coordinates), relative location information (such as distance or topological relationship), etc. Therefore, a central task of the spatial data model is to research the expression of the concept of spatial entity and spatial relationships. The research content is the basis of the design concept of spatial database as well as construction modes, and it directly affects the effectiveness of spatial database organization, spatial query, spatial reasoning and spatial analysis.

To a great extent, the understanding and research of 3D spatial data model determine the development and application of scientific computing visualization system. Many scholars carried out extensive exploration and research work. However, because of the hugeness and complexity of 3D spatial data, researchers have not achieved a consensus on the problem of three-dimensional data model so far. There are many data models about the three-dimensional entity and their mutual relationships, and in some areas or projects, the data model shows advantages, but it is almost impossible if we want to use a generic model to describe and express the objective world of all three-dimensional entities and their spatial relationships. Three-dimensional vector data structure, which was put forward by Yuanqing Lee, can be more reasonable to describe the slope geological entity. This structure has obvious advantages and the author used this theory to set up the three-dimensional slope model.

\subsection{Topological Relation}

How to establish and maintain the space element topological relationship is a core issue in the field of scientific computing visualization research [4]. The establishment of the topological relationship makes all kinds of space operation and information queries easy to implement. However, the establishment of 3D topology relationship in scientific computing visualization is an intractable problem, because the structure of the research aim is extremely complex and irregular. In the practical application of geotechnical engineering in scientific computing visualization research, complex features can be described by various body full of space domains, the domain of body surfaces, the boundary of the ring's surface, arcs of the ring and nodes on the arc. In general, the body domain is the basic building block of target entities, and we can say that any complex entity is composed of a domain of the body (natural or artificial). The concept of body, plane, line and point is dynamic, and in different scales or different research focus, they can transform into each other.

\subsection{Model Building}

The three-dimensional computer simulation and visualization study on the rock mass as well as the establishment of three-dimensional model of rock mass can help to research and analyze the form of rock mass and its changing rule more intuitively. When establishing a three-dimensional model of rock mass, we should start from creating the space position of the drilling, which provides the basis for the scientific computing visualization model of rock mass. Then, starting from the profile, according to the existing data, we can use the projection shape of rock mass in the prospecting line section, and build rough geometrical shape of the space. In the final phase, the three-dimensional model of the ore body can be formed. At this point, we can operate the model by rotation, amplification and narrowing, and analyze the distribution law of rock mass from three-dimensional space forms, and the form and spatial changes of the rock mass. In mining engineering based on the research of the ore body 3D model, the ore body 3D model is set up, not only to give a new understanding of ore bodies, but also to help change the traditional graphic thinking mode. The study perspective of the spatial distribution law of ore bodies is from the angle of three-dimensional space. It provides an intuitive and advanced method for the study of geology and mine production.

\section{3D Numerical Simulation Analysis of Rock Mass}

In geotechnical engineering, rock mass is in the three-dimensional stress state, which has an important effect on the stability of the slope. At the same time, because of the influence of slope excavation, blasting vibration and other factors, the structure and stress of the rock mass are changing constantly. In the research of the stability of geotechnical engineering, this problem was studied as a two-dimensional problem, in which there are a large number of simplified conditions, making it impossible to consider the three directions of principal stress situation at the same time and making 
the result of calculation unable to correspond to reality. In order to reflect the actual situation and illustrate the three principal stress effects on the stability of geotechnical engineering more truly, this problem must be analyzed as a three-dimensional problem [5].

In 3D numerical simulation analysis, at the first stage, according to the sliding body position, we should establish physical and mechanical models, and set up a three-dimensional physical mechanics model on the basis of landslide topography and structure characteristics. The second stage is to split 3D model into unit body which needs to be calculated, and use the basic principle of finite element method and calculation method to calculate and analyze it. In finite element analysis, visualization technology is applied to implement the mesh generation and graphical display of the finite element result data, which is the so-called before and after treatment of finite element analysis. Existing finite element analysis software can display the two-dimensional graphics well, but its visualization function of three-dimensional data field is weak. However, data fields, including engineering calculation data and measurement data, which are formatted by scientific calculation, are often three-dimensional space data fields, namely a geometric location of the space where the data values are defined one by one. Therefore, the expression of the visual image of finite element analysis result data is one of the important aspects of scientific computing visualization technology research. Numerical calculation method, known as a finite element method, discrete element method (DEM), discontinuous deformation analysis (DDA) and so on, also plays an important role in geotechnical engineering, due to the fact that rock mass is different from other homogeneous solid bodies, especially for the reason that rock mass has many faults, interfaces or joints in most cases. Therefore, it is also known as the joint rock mass. The computational models of DEM and DDA are based on the principle that the rock mass is divided into isolated blocks according to their respective joints or interfaces, and its calculation model is composed of all integral blocks. It is obvious that the 3D feature of this rock system is clear, which is divided according to the real faults, joints, and interfaces in the space. From this point, in numerical analysis of DEM and DDA, the analysis of 3D is much more meaningful and valuable than that of $2 \mathrm{D}$, and only in the analysis of 3D can calculation analysis method of DEM and DDA and this backbone block model obtain reasonable results, and get approvals by engineering. In addition to modeling, three-dimensional numerical simulation analysis should also have powerful post-processing functions, such as using visualization technology to analyze the results, or generating various forms of three-dimensional contour maps, chromatogram, vector graphics, etc. In the study of rock mass stability analysis method, the three-dimensional numerical simulation analysis method of three-dimensional rock mass is a simple and practical analysis method with higher precision. The graphics display of geological entity scientific computing visualization allows the geological workers to see the geometry relationship and distribution of geological phenomenon more intuitively, and analyze mechanical calculation more accurately.

\section{The Scientific Computing Visualization Research of Rock Mass Structure}

All kinds of geological information of rock discontinuous structural plane are obtained from the field measuring instrument or observation. However, the information is usually discrete data, and it is difficult for geological personnel to use these data directly for the analysis of the structure plane in rock mass distribution or the intersection relationship between each other. Facing these valuable data, geological engineers will surely raise a question about the way to use these data to infer the development of structural plane, the distribution in the studied area and spatial combination relations. In the past, geological personnel often used graphics to describe their understanding of geologic body, and now they tend to use computers to display the information automatically in the distribution of natural rock mass. The rock mass structure determines rock mechanics properties and is also the basis of the analysis of rock mass in engineering, so the formation of the rock mass structure is vital. It is true that rock fractures exist in the state of three dimensions. In order to simplify the calculation, the rock mass structure is described by a two-dimensional network in the past, but with the deepening of the research work, the theoretical calculation has developed into a three-dimensional structure from the two-dimensional structure before, and rock mass damage fractures described in three dimensions are more accurate. For three-dimensional fracture network simulation and the generation of rock 
mass, the crucial point is the acquisition and analysis of rock fracture parameters. Commonly used method is to measure two-dimensional cracks in artificial measurement methods, such as measuring the length of the fracture trace or wide gaps, calculating the number of fractures and measuring the angles of fractures. The probability and statistics modal analysis of the statistics data of the fracture's two-dimensional occurrence is helpful in generating a three-dimensional network.

In today's geological application technology, the use of graphics and images on screen is an effective method of describing rock mass structure information. Through the scientific computing visualization graphics display of the structural plane, geological engineers can see surface geometry, the distribution and relationship of rock mass structural plane space intuitively, and also make accurate scientific analysis, thereby making reasonable and scientific conclusions and working out solutions to geological problems.

\section{Conclusion}

Because scientific computing visualization technology has great efficiency and visibility for large data processing, the application of such technology in geotechnical engineering research is of great significance. With the further research in the field of geotechnical engineering, visualization technology applied in this field will constantly increase. This paper mainly describes the application of scientific computing visualization technology in three-dimensional modeling of rock mass, three-dimensional numerical simulation analysis of rock mass and the three-dimensional network of rock mass structure simulation research, to show that visualization technology is helpful in geotechnical engineering research.

\section{References}

[1] Moore, R. R., \& Johnson, S. E. (2001). Three-dimensional reconstruction and modelling of complexly folded surfaces using Mathematica. Computers \& Geosciences, 27(4), 401-418.

[2] Marschallinger, R. (2001). Three-dimensional reconstruction and visualization of geological materials with IDL_-examples and source code. Computers \& Geosciences, 27(4), 419-426.

[3] Wu, Q., Xu, H., \& Zou, X. (2005). An effective method for 3D geological modeling with multi-source data integration. Computers \& Geosciences,31(1), 35-43.

[4] Tolosana-Delgado, R., Pawlowsky-Glahn, V., \& Egozcue, J. J. (2008). Simplicial indicator kriging. Journal of China University of Geosciences,19(1), 65-71.

[5] Tanagho, Y. S., Andriole, G. L., Paradis, A. G., Madison, K. M., Sandhu, G. S., Varela, J. E., \& Benway, B. M. (2012). 2D versus 3D visualization: impact on laparoscopic proficiency using the fundamentals of laparoscopic surgery skill set. Journal of Laparoendoscopic \& Advanced Surgical Techniques, 22(9), 865-870. 